DrAfT VERSION OCTOBER 30, 2020

Typeset using IATEX RNAAS style in AASTeX62

\title{
Predictions for strong lens detections with the Nancy Grace Roman Space TELESCOPE
}

\author{
Charles Weiner, ${ }^{1}$ Stephen Serjeant, ${ }^{1}$ and Chris Sedgwick ${ }^{1}$ \\ ${ }^{1}$ School of Physical Sciences, The Open University, Milton Keynes MK7 6AA, UK
}

\begin{abstract}
Strong gravitational lensing is an ideal tool for mapping the distribution of dark matter and for testing the values of cosmological parameters. Forthcoming surveys such as Euclid and the Rubin Observatory LSST should increase the number of known strong lensing systems significantly, with for example over 100,000 such systems predicted in the Euclid wide survey. In this short research note we predict that approximately 17,000 strong gravitational lenses will also be detectible in the Nancy Grace Roman Space Telescope 2000 square degree survey, using the LensPop gravitational lensing model. We present predicted distributions in source and deflector redshifts, magnitudes, and magnifications. Although this survey is not primarily designed as a strong lensing detection experiment, it will still provide a large complementary catalogue to shallower, wider-area forthcoming lensing discovery projects.
\end{abstract}

Keywords: infrared astronomy — gravitational lenses — cosmology observations — galaxy evolution

\section{INTRODUCTION}

Gravitational lensing depends only on the distribution and geometry of foreground matter, and is independent of its composition or luminosity. It is therefore an ideal tool for mapping the distribution of dark matter and testing the values of cosmological parameters. In addition, magnification by the lens can enhance the spatial resolution of background galaxies by $1-2$ orders of magnitude.

Strong gravitational lenses have been detected in various previous and ongoing surveys. The Sloan Lens ACS Survey (SLACS; Bolton et al. 2006) combined the massive data volume of the Sloan Digital Sky Survey (SDSS) with the highresolution imaging capability of the Hubble Space Telescope to identify and study some 100 lenses and lens candidates (Auger et al. 2009). Neural networks are increasingly used to identify lenses in data catalogues: for example, a recent study of the Dark Energy Survey (DES) using convolutional neural networks found 84 strong lens candidates (Jacobs et al. 2019). Citizen science is also being used to find lenses which are not always clear to automated searches (Marshall et al. 2016). These studies (and serendipitous discoveries) have brought the total number of strong lenses discovered to date to several hundred.

Forthcoming surveys should increase this number significantly. The Large Synoptic Telescope Survey (LSST; Abell et al. 2009, Ivezic et al. 2008) will start observing in 2022. Also highly significant for the discovery of strong lenses will be two space-borne instruments: Euclid (Laureijs et al. 2011), a European Space Agency project planned to launch in 2022, and the Nancy Grace Roman Space Telescope, formerly known as the Wide Field InfraRed Survey (WFIRST; Green et al. 2012), a NASA mission planned to launch in 2025.

Two detailed predictions of strong lensing have already been made for Euclid: Serjeant (2014) presented selection techniques to reliably identify up to 10,000 strong gravitational lenses using Euclid's spectroscopic surveys, and the gravitational lens model LensPop developed by Collett (2015) predicted 170,000 strong lenses to be detectable in Euclid's wide imaging survey. This paper presents the first prediction of detectable strong lenses for the Roman Space Telescope, based on the LensPop model.

Corresponding author: Chris Sedgwick

chris@sedgwick.uk.net 


\section{METHODOLOGY}

The LensPop model is described in Collett (2015) with its source code available as open source software ${ }^{1}$. The model has required only minor modifications to extend it to the Roman Space Telescope.

The model assumes strong lensing by elliptical galaxies, modelled as singular isothermal ellipsoids (SIEs). Firstly, a population of foreground SIEs is generated with five key parameters: redshift, stellar velocity dispersion, flattening, effective radius and absolute magnitude. Secondly, the galaxy population simulated for the LSST collaboration (Connolly et al. 2010) is assumed for background sources. The lensing cross-section of the foreground population is then projected onto the background source populations to generate an idealised set of lens systems (deflector + source). Finally, the model applies the observing parameters of the survey being considered to discover the final set of strong lenses detectable by that survey.

Four criteria are used for accepting the lens: the source is within the Einstein radius; the image is resolved; tangential shearing of arcs is detectible; the signal-to-noise ratio is over 20 :

$$
\begin{gathered}
\theta_{E}^{2}>x_{s}^{2}+y_{s}^{2} \\
\theta_{E}^{2}>r_{s}^{2}+(s / 2)^{2} \\
\mu r_{s}>s, \mu>3 \\
\mathrm{SNR}>20
\end{gathered}
$$

where $\theta_{E}$ is the Einstein radius, $\left(\mathrm{x}_{s}, \mathrm{y}_{s}\right)$ are the coordinates of the centre of the source with respect to the lens, $\mathrm{r}_{s}$ is the source radius, $\mathrm{s}$ is the point spread function full width half maximum (seeing, in ground-based contexts), $\mu$ is the total magnification and SNR is the signal-to-noise ratio of the lensed residual.

\section{APPLICATION TO THE ROMAN SPACE TELESCOPE}

The Roman Space Telescope will have six filters, but our work is based on the J band (referred to by its midpoint as J_129), which has the greatest AB magnitude depth of 26.9. The survey parameters added to the code included survey area $2000 \mathrm{deg}^{2}$, point spread function full width half maximum of $0.12^{\prime \prime}$ and sky brightness 23.5 mags $\operatorname{arcsec}^{-2}$.

Another modification to the code was made to produce values for source and lens J_129 magnitudes. An extrapolation was made from SDSS values for $\mathrm{i}$ and $\mathrm{z}$ bands: the wavelength difference between the $\mathrm{i}$ and $\mathrm{z}$ bands is $\simeq 0.1 \mu \mathrm{m}$, and between $\mathrm{z}$ and $\mathrm{J}$ bands is $\simeq 0.44 \mu \mathrm{m}$, so a simple log-linear extrapolation gives $m_{J}=m_{z}-\left(m_{i}-m_{z}\right) * 4.4$. We use this phenomenological extrapolation over a short wavelength range to minimise the assumptions about galaxy SEDs.

The Roman Space Telescope survey is intended to cover $2000 \mathrm{deg}^{2}$ over a five year period, so each sky position is expected to have several hundred seconds integration time. We have estimated an effective exposure time of 700 seconds in the model. For this value, we verified that a sky brightness of 23.5 mags $\operatorname{arcsec}^{-2}$ is consistent with a $5 \sigma$ point source sensitivity of magnitude $\sim 26.9 \mathrm{AB}$ in the J_129 band in simulated lens-subtracted images.

This work assumes $H_{0}=70.0 \mathrm{kms}^{-1} \mathrm{Mpc}^{-1}, \Omega_{M}=0.3$ and $\Omega_{\Lambda}=0.7$. Further details are given in Weiner (2019).

\section{RESULTS}

The model predicts 16,778 detectable strong lenses for the Roman Space Telescope. Histograms of key predictions are shown in in Figure 1: lens redshifts (mean 0.6 \pm 0.3 ), source redshifts (mean 1.9 \pm 0.9 ), magnification (mean 5.5 \pm 3.9 ), velocity dispersion of lens (mean 219 \pm 50 ), Einstein radius (mean $0.8^{\prime \prime} \pm 0.4^{\prime \prime}$ ) and source magnitude (mean 24.8 \pm 1.6 ). A typical image of a predicted lens system, and the image minus the lens are also in Figure 1.

The predicted average magnification is lower than for Euclid (5.5 compared to 7.2), although with a smaller variance. Predicted mean redshifts for both lenses and sources are similar in each case. For comparison, the model predicts 39,000 strong lenses will be detectable by the LSST.

\footnotetext{
${ }^{1}$ https://github.com/tcollett/LensPop
} 


\section{DISCUSSION AND SUMMARY}

The model we have used is based on simulated data and an assumption that SIEs account for most lens systems. For the background sources, the LSST simulated source catalogue is limited in depth, so the model may be underpredicting the number of highly magnified sources. On the other hand, sources as faint as the limiting magnitude of 26.9 are likely to be extended sources and so may not be detected since the flux will be spread over several pixels. Actual lens discoveries will depend on the methods developed to identify such large numbers of lenses in this survey.

It would seem that a significant increase, potentially up to two orders of magnitude, of strong lenses available for further study will be provided by the two new space telescopes. This will provide the opportunity for a great deal of new science on dark matter, cosmological parameters and on early galaxy formation from the distant sources discovered, giving key targets for follow-up observations.
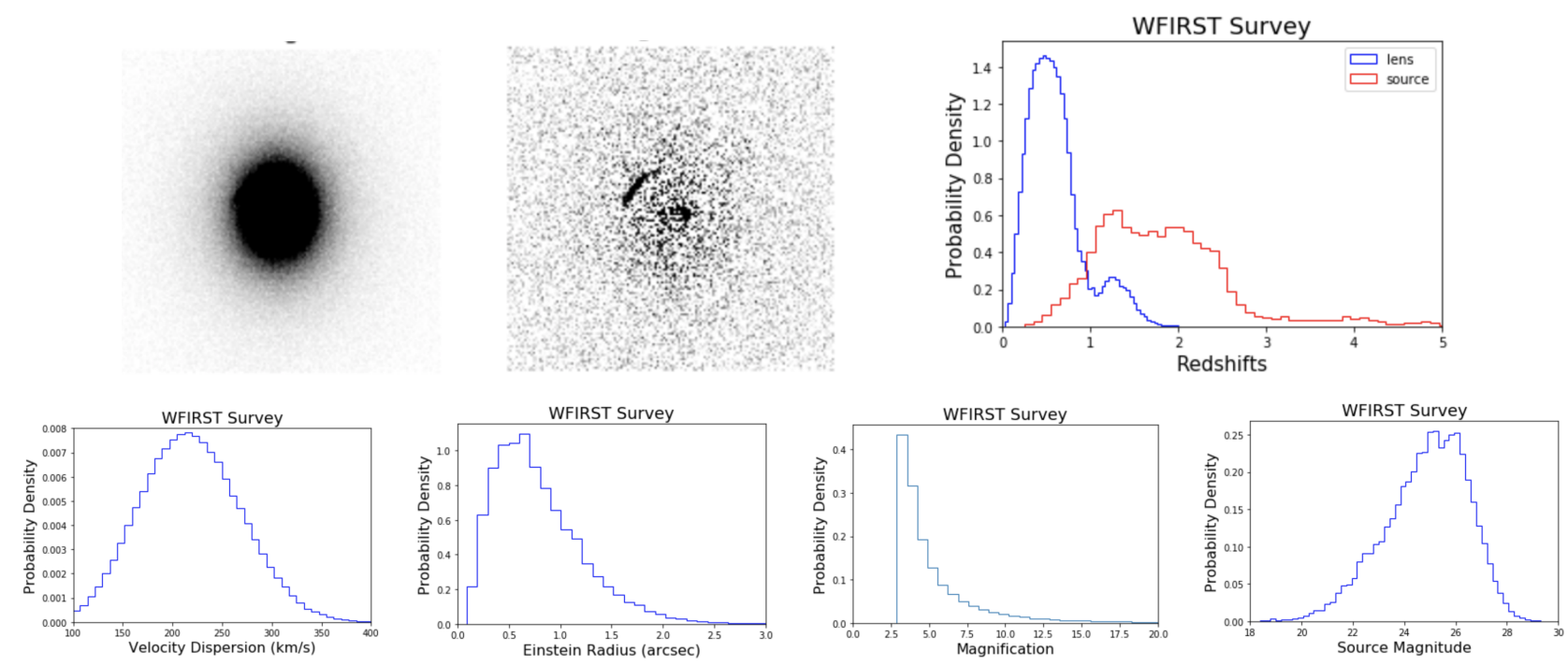

Figure 1. Image of a simulated lens detectable by the Roman Space Telescope in the J_129 filter band; image minus lens (assuming subtraction is Poisson noise limited); histograms of distributions of predicted redshifts; the velocity dispersion of the lens; the Einstein radius; the distribution of magnifications; and source magnitudes.

We thank Tom Collett, the Project Scientists in the WFIRST Communications Team and Judith Croston. SS thanks STFC for support grant ST/P000584/1. 


\section{REFERENCES}

Abell, P.A., Burke, D.L., Hamuy, M. et al. 2009, LSST science book v2.0, Technical report

Auger, M., Treu, T., Bolton, A. et al. 2009, ApJ, 705, 1099

Bolton, A.S., Burles, S., Koopmans, L.V. et al. 2006, ApJ, 638, 703

Collett, T.E., 2015, ApJ, 811, 20

Connolly, A.J., Peterson, J., Jernigan, J.G. et al. 2010, Proc. SPIE, 7738, 7738IO

Green, J., Schechter, P., Baltay, C. et al. 2012, arXiv 1208.4012
Ivezic, Z., Tyson, J., Abel, B. et al. 2008, arXiv 0805.2366 Jacobs, C., Collett, T., Glazebrook K. et al. 2019, MNRAS, 484,5330

Laureijs, R., Amiaux, J., Arduini, S. et al. 2011, arXiv 1110.3193

Marshall, P.J., Verma, A., More, A. et al. 2016, MNRAS, 455,1171

Serjeant, S., 2014, ApJ, 793, L10

Weiner, C.F., 2019, MPhil thesis (The Open University) 